\title{
Inhalt des ersten Bandes
}

Zu dieser Ausgabe . . . . . . . . . . . . . . . . . . . XV

ERSTER TEIL

Das Geld: Seine Herrschaft in Friedenszeiten und sein Zusammenbruch während des Krieges ～. . . . 1

Vorwort des Verfassers . . . . . . . . . . . . . . . . . . . . . . . . . . . . . . 3

Teil I

Die Herrschaft des Geldes in der Friedenswirtschaft . . . . . . . . . . . . . . . . . . . . 5

1. Das Geld - ein gesellschaftliches Produkt . . . . . . . . . . . . . . . . . . . . . 5

2. Geld als Maß der Werte und Maßstab der Preise . . . . . . . . . . . . . . . . . . . . . 8

3. Die Rolle des Staates bei der Entstehung des Geldes . . . . . . . . . . . . . . . . . . . . 10

4. Geldzeichen als Ersatz für wirkliches Geld . . . . . . . . . . . . . . . . . . . . . . . . 14

5. Die in Umlauf befindliche Geldmenge . . . . . . . . . . . . . . . . . . . . . . . . . . 17

6. Die Rolle des Geldes in der warenproduzierenden Gesellschaft . . . . . . . . . . . . . . . 20

7. Banknoten . . . . . . . . . . . . . . . . . . . . . . . 25

8. Das Geldsystem Ungarns . . . . . . . . . . . . . . . . . . . . . . . . . . . . . . . . 28

9. Das Weltgeld . . . . . . . . . . . . . . . . . . . . . . . . . . . . . 31

10. Die Verzinsung des Geldes, die Banken. . . . . . . . . . . . . . . . . . . . . . . . . . 35

11. Geldmangel und Geldüberschuß . . . . . . . . . . . . . . . . . . . . . . . . . 41

12. Die Stabilisierung des Geldwertes durch die Emissionsbanken . . . . . . . . . . . . . . . . 44

Teil II

Das Geld während des Krieges . . . . . . . . . . . . . . . . . . . . . . . . . . . . . 47

1. Der Rückgang der Kaufkraft des Geldes während des Krieges. . . . . . . . . . . . . . . . 47

2. Der Warenmangel während des Krieges, Teuerung und Spekulation . . . . . . . . . . . . 49

3. Die Verselbständigung des Metallgeldes und der Banknoten während des Krieges . . . . . . . 53

4. Die Geld,,tragödie“" während des Krieges . . . . . . . . . . . . . . . . . . . . . . . . . 57

5. Das Disagio des Geldes in Kriegszeiten. . . . . . . . . . . . . . . . . . . . . . 61

6. Das Ende der Herrschaft des Geldes . . . . . . . . . . . . . . . . . . . . . . . . 66

ZWEITER TEIL

Die wirtschaftspolitischen Probleme der proletarischen Diktatur. . . . . . . . . . . . . . . . 72

Vorwort zur ersten Auflage. . . . . . . . . . . . . . . . . . . . . . . . . . . . . . . . .73

1. Die Krise des Kapitalismus . . . . . . . . . . . . . . . . . . . . . . . . . . . . . . . 77

2. Das Problem der Steigerung der Produktion . . . . . . . . . . . . . . . . . 85

3. Rückgang der Lebenshaltung des industriellen Proletariats zu Beginn der proletarischen Diktatur 95

4. Die Expropriation der Expropriateure . . . . . . . . . . . . . . . . . . 103

5. Die Organisationsprobleme der proletarischen Volkswirtschaft. . . . . . . . . . . . . 110

6. Das Problem der Arbeitsdisziplin und der Arbeitsintensität . . . . . . . . . . . . . . . . . 121

7. Das Problem der Beamtenschaft . . . . . . . . . . . . . . . . . . . . . . . . . . . 127

8. Die Agrarpolitik des proletarischen Staates . . . . . . . . . . . . . . . . . . . . . . . . . 134

9. Die Lebensmittelversorgung. . . . . . . . . . . . . . . . . . . . . . . . . . . . . . . 141 
10. Das Problem der Güterverteilung . . . . . . . . . . . . . . . . . . . . . . 148

11. Das Geldproblem in der Diktatur des Proletariats . . . . . . . . . . . . . . . . . . 156

12. Der proletarische Staatshaushalt . . . . . . . . . . . . . . . . . . 164

13. Internationale Wirtschaftsprobleme in der proletarischen Diktatur . . . . . . . . . . . 169

Schlußwort. . . . . . . . . . . . . . . . . . . . . . . . 175

DRITTER TEIL

Die Krise der kapitalistischeq Weltwirtschaft ～. . . . . . . . . . . . . . . . . . . . . . . 177

Vorwort zur zweiten Auflage . . . . . . . . . . . . . . . . . . . . . . . . 181

Vorwort zur ersten Auflage . . . . . . . . . . . . . . . . . . . . . . . 183

I. Die Veränderungen der Weltwirtschaft durch den Krieg _ . . . . . . . . . . . . . . . 184

1. Die Grundtatsachen der Weltwirtschaft vor dem Kriege . . . . . . . . . . . . . . . 184

2. Verschiedene Folgen des Weltkrieges auf verschiedene Länder . . . . . . . . . . 185

a) Kriegführende Länder . . . . . . . . . . . . . . . . . . . . . . . 185

b) Die neutralen kapitalistischen Länder . . . . . . . . . . . . . . . . . . 191

c) Die Industrialisierung der Kolonialländer. . . . . . . . . . . . . . . . . . 203

II. Die Hochkonjunktur nach Kriegsende . . . . . . . . . . . . . . . . . . . . . . . . . 206

III. Die gegenwärtige Wirtschaftskrise . . . . . . . . . . . . . . . . . . . . . . . . . . . 214

Die Ursachen der gegenwärtigen Weltkrise . . . . . . . . . . . . . . . 215

1. Absolute Unterproduktion und relative Uberproduktion . . . . . . . . . . . . . 217

2. Verschiedene Preisentwicklung der landwirtschaftlichen und industriellen Waren . . . . 220

3. Nichtanpassung der Produktion an die veränderten Einkommensverhältnisse . . . . . . . 224

4. Die Störung der weltwirtschaftlichen Arbeitsteilung durch Industrialisierung bisher agrarischer Länder . . . . . . . . . . . . . . . . . . . . . . . . . . . 226

Krise und Arbeitslosigkeit . . . . . . . . . . . . . . . . . . . . . . . . . 227

IV. Die Lebenshaltung der Arbeiterklasse seit Kriegsausbruch. . . . . . . . . . . . . . . . . 230

1. Deutschland und die mitteleuropäischen Staaten . . . . . . . . . . . . . . . . . 230

2. Ententeländer . . . . . . . . . . . . . . . . . . . . . 234

V. Die Arbeitsleistung seit Kriegsbeginn . . . . . . . . . . . . . . . . . . . 239

VI. Das Krisenjahr 1921 . . . . . . . . . . . . . . . . . . . . . . . . . . . . . . . . . 242

a) Die Produktion im Jahre 1921 verglichen mit $1920 \ldots \ldots$. . . . . . . . . . . . . . 242

b) Die Arbeitslosigkeit im Durchschnitt des Jahres 1921 . . . . . . . . . . . . . . . . 243

c) Das Auseinanderfallen der Weltwirtschaft . . . . . . . . . . . . . . . . . . . . 243

d) Das Zusammenschrumpfen des Außenhandels . . . . . . . . . . . . . . . . . . . . 244

e) Die Preisbewegung im Jahre $1921 \ldots \ldots$. . . . . . . . . . . . . . . . . . 245

VII. Symptome der Besserung der Konjunktur während des Jahres $1921 \ldots$. . . . . . . . . . 247

VIII. Tendenzen und Bestrebungen zur Überwindung der Weltwirtschaftskrise . . . . . . . . . . 250

1. Die immanenten Überwindungstendenzen in der Weltwirtschaft . . . . . . . . . . . 250

2. Die Reparationsfrage . . . . . . . . . . . . . . . . . . . . . . . . . . . 251

3. Tendenzen zur Ausgleichung der Preise landwirtschaftlicher und industrieller Produkte 255

4. Anpassungstendenzen an die veränderte Einkommensverteilung . . . . . . . . . 255

5. Die Überwindung der überflüssigen Industrialisierung. . . . . . . . . . . . . 257

6. Tendenz zur Überwindung der Krise durch Kapitalexport . . . . . . . . . . . . . . 257

IX. Bewußte Bestrebungen zur Wiederhersteliung des weltwirtschaftlichen Gleichgewichts . . . . 260

1. Abbau der weltwirtschaftlichen Arbeitsteilung . . . . . . . . . . . . . . . . . . 260

2. Erschließung neuer Märkte: das Problem China . . . . . . . . . . . . . . 263

3. Wiederaufrichtung der Unterproduktionsgebiete in Europa . . . . . . . . . . 265 
X. Die Krise Rußlands und die Weltwirtschaftskrise . . . . . . . . . . . . . . . . 268

XI. Überwindungsversuche auf Kosten des Proletariats. . . . . . . . . . . . . . . 273

XII. Vom kommenden Weltkrieg. . . . . . . . . . . . . . . . . . . . 275

XIII. Die Bilanz der drei Nachkriegsjahre . . . . . . . . . . . . . . . . . . . . 278

VIERTER TEIL.

Die Niedergangsperiode des Kapitalismus . . . . . . . . . . . . . . . . . . . . . . . . . . 281

Vorwort . . . . . . . . . . . . . . . . . . . . . . . . . 283

1. Kapitel

Aufstieg und Niedergang des Kapitalismus . . . . . . . . . . . . . . . . . . . . . . . . . 284

2. Kapitel

Das Wesen des Niederganges des Kapitalismus . . . . . . . . . . . . . . . . . . . . . . . . 287

3. Kapitel

Die Rolle des Krieges im Kapitalismus. . . . . . . . . . . . . . . . . . . . . . . . . . . . 298

4. Kapitel

Die Wirtschaftstypen der Niedergangsperiode des Kapitalismus . . . . . . . . . . . . . . 301

5. Kapitel

Die Wirtschaftsentwicklung des letzten Jahres. . . . . . . . . . . . . . . . . . . . . . 310

6. Kapitel

Entwicklungstendenzen und Zukunftsaussichten . . . . . . . . . . . . . . . . 319

FÜNFTER TEIL

Wirtschaft und Wirtschaftspolitik im 4. Vierteljahr 1929. . . . . . . . . . . . . . . . . . . . . 324

I. Krise in den Vereinigten Staaten - Krise der Weltwirtschaft . . . . . . . . . . . 325

1. Verlorene Illusionen . . . . . . . . . . . . . . . . . . . . . . . . 325

2. Die Ursachen der Krise in den Vereinigten Staaten von Amerika . . . . . . . . . . . 327

3. Die bereits erreichte Tiefe der Krise . . . . . . . . . . . . . . . . . . . . . . . 330

4. Börsenhausse und Börsenkrach als Krisenelemente . . . . . . . . . . . . . . . . 332

5. Organisierte Krisenbekämpfung - ein aussichtsloser Versuch . . . . . . . . . . . . 339

6. Die Demagogie Fords . . . . . . . . . . . . . . . . . . . . . . . . . . . . 343

7. Modifikation des Krisenverlaufs infolge des monopolistischen Charakters des Kapitalismus der Vereinigten Staaten von Amerika . . . . . . . . . . . . . . . . . . . . . . . . 344

8. Die Krise in den Vereinigten Staaten führt zu einer Krise in allen kapitalistischen Ländern $\mathbf{3 4 6}$

9. Verschärfter Kampf um den Weltmarkt: erhöhte Kriegsgefahr . . . . . . . . . . . . . 349

10. Zuspitzung der inneren Gegensätze; Herannahen der proletarischen Revolution . . . . 354

II. Die Schranken des Aufstiegs des deutschen Kapitalismus . . . . . . . . . . . . . . . . . 357

Die Gestaltung der Produktion . . . . . . . . . . . . . . . . . . . . . . . . . 358

Mißverhältnis zwischen Produktion und Produktionskapazität . . . . . . . . . . . . 361

Das Verwertungsproblem des deutschen Kapitalismus . . . . . . . . . . . . . . . . . 365

KapitalüberfluB - Kapitalmangel . . . . . . . . . . . . . . . . . . . . . . . . 370

III. Allgemeiner Teil . . . . . . . . . . . . . . . . . . . . . . . . . . . . 376

Das allgemeine Bild der Weltwirtschaft . . . . . . . . . . . . . . . . . . . 376

Die Krise und das Preisproblem . . . . . . . . . . . . . . . . . . . . . . . . . . . . . 378

Geld- und Kapitalmarkt . . . . . . . . . . . . . . . . . . . . . . . . . . . . . . . 382

Abwehrversuche gegen die Konkurrenz der USA . . . . . . . . . . . . . . . . . . . 384 
Die Arbeitslosigkeit . . . . . . . . . . . . . . . . . . . . . . . . . 385

Die zweite Haager Konferenz . . . . . . . . . . . . . . . . . . . . . . . . . 388

IV. Spezieller Teil . . . . . . . . . . . . . . . . . . . . . . . . . 391

Deutschland . . . . . . . . . . . . . . . . . . . . 391

Frankreich . . . . . . . . . . . . . . . . . . . . . . . . . . 398

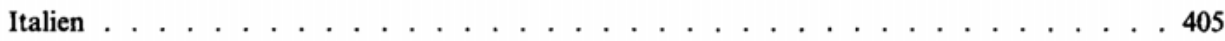

England . . . . . . . . . . . . . . . . . . . . . . . . 409

Vereinigte Staaten von Amerika . . . . . . . . . . . . . . . . . . . . . . . . . 419

SECHSTER TEIL

Die historischen Wurzeln der Besonderheiten des deutschen Imperialismus . . . . . . . . . . . . . 427

Die verspätete Entwicklung des deutschen Industriekapitalismus. . . . . . . . . . . . . . . . . 427

Der preußische Weg in der Landwirtschaft . . . . . . . . . . . . . . . . . . . . . 430

Die Vorherrschaft Preußens in Deutschland und das Preußentum . . . . . . . . . . . . . .433

Der deutsche Imperialismus der Kaiserzeit . . . . . . . . . . . . . . . . . . . . 435

Der deutsche Imperialismus: Weimar und der Aufstieg des Nationalsozialismus . . . . . . . . . 440

Der deutsche faschistische Imperialismus . . . . . . . . . . . . . . . . . . . . . . .442

Sachregister . . . . . . . . . . . . . . . . . . . . . . . 449

Personenregister . . . . . . . . . . . . . . . . . . . . 457 


\section{Inhalt des zweiten Bandes}

ERSTER TEIL

\section{Die Weltwirtschaftskrisen}

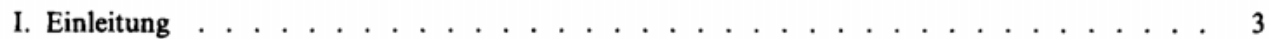

II. Die Krisen und der Niedergang des Kapitalismus . . . . . . . . . . . . . . . . . . 6

III. Die Krisentheorie von Marx . . . . . . . . . . . . . . . . . . . . . . . . . . . . . 9

IV. Allgemeine Charakteristik der Krisen in den einzelnen Perioden des Kapitalismus . . . . . . 13

Zyklen und Krisen in der ersten Hälfte des 19. Jahrhunderts . . . . . . . . . . . . . . 15

Zykien und Krisen im dritten Viertel des 19. Jahrhunderts . . . . . . . . . . . . . . . 16

Die Agrarkrise des 19. Jahrhunderts und ihr Einfluß auf die industriellen Zyklen (1873 bis

1895) . . . . . . . . . . . . . . . . . . . . 19

Der Einfluß des Kapitalexports auf Krisen und Zyklen . . . . . . . . . . . . . . . . 23

Zyklen und Krisen des Monopolkapitalismus in der Vorkriegszeit . . . . . . . . . . . 24

V. Krisen und Zyklen in der allgemeinen Krise des Kapitalismus . . . . . . . . . . . . . . . 27

Die Rüstungskonjunktur . . . . . . . . . . . . . . . . . . . . . . . . . . . . . . 29

Die Krise von 1920. . . . . . . . . . . . . . . . . . . . . . . . . . . . . . . . . 30

Die zeitweilige Stabilisierung und der Zyklus 1921-1929 . . . . . . . . . . . . . . . 31

Die Krise von 1929. . . . . . . . . . . . . . . . . . . . . . . . . . . . . . . . . 32

Die Agrarkrise. . . . . . . . . . . . . . . . . . . . . . . . . . . . . . . . . . 38

Neuer Zyklus · Depression · Perspektiven . . . . . . . . . . . . . . . . . . . . . 46

Krisenfreie Entwicklung der sozialistischen Wirtschaft in der UdSSR . . . . . . . . . . 49

ZWEITER TEIL

Konjunkturforschung und Krisentheorie . . . . . . . . . . . . . . . . . . . . . 55

Die Leistungen der Konjunkturforschung . . . . . . . . . . . . . . . . . . . . . . . . . 60

a) Die Beschreibung des Konjunkturganges . . . . . . . . . . . . . . . . . 60

b) Die Ergebnisse der Messungen des Wirtschaftsverlaufes . . . . . . . . . . . . . . . . 63

DRITTER TEIL

Studien über Grundzïge der Entwicklung imperialistischer Hauptländer . . . . . . . . . . . . . . 70

I. Die Krise der englischen Wirtschaft: eine Teilerscheinung des Niederganges des Kapitalismus 71

a) Der Entwicklungsgang der britischen Wirtschaft . . . . . . . . . . . . . . . . . 72

b) Inflation und Deflation als Weltwirtschaftsfaktoren . . . . . . . . . . . . . . . . 77

c) Das Problem der englischen Bourgeoisie: Die Last der Rente . . . . . . . . . . . . . 81

d) Das Problem der deutschen Bourgeoisie: Die Rationalisierung . . . . . . . . . . . . . . 86

e) Kein Aufstieg, sondern verschärfte Kämpfe . . . . . . . . . . . . . . . . . . . . . . . . 90

f) Amerika kann keine Abhilfe schaffen . . . . . . . . . . . . . . . . . . . . . . . . . . . 94

II. Der Weg des deutschen Kapitalismus. . . . . . . . . . . . . . . . . . . . . . . . . . . 99

1. Die Grundtatsachen der deutschen Wirtschaft . . . . . . . . . . . . . . . . . . . 100

2. Der Ausbau des sachlichen Produktionsapparates . . . . . . . . . . . . . . . . . 105

3. Produktionsfähigkeit und tatsächliche Produktion . . . . . . . . . . . . . . . . . 107 
4. Die Gestaltung des Volkseinkommens . . . . . . . . . . . . . . . . . . . 110

5. Die Reparationslasten und Auslandsanleihen . . . . . . . . . . . . . . . . . . . . 112

6. Die zwei Auswege: Stärkung des inneren Marktes oder erhöhter Auslandsabsatz . . . . . . 117

7. Die Gestaltung des deutschen Außenhandels . . . . . . . . . . . . . . . . 119

8. Zusammenfassung . . . . . . . . . . . . . . . . . . 125

III. Der Weg des französischen Kapitalismus . . . . . . . . . . . . . . . . . . . . . . . . 127

Die Entwicklung der Bevölkerung . . . . . . . . . . . . . . . . . . . . . . 127

Die Entwicklung der französischen Landwirtschaft . . . . . . . . . . . . . . . . . 128

Bergbau und Industrie . . . . . . . . . . . . . . . . . . . . . . . . . . 130

Der Bergbau . . . . . . . . . . . . . . . . . . . . . . . 132

Die industrielle Produktion . . . . . . . . . . . . . . . . . . . . 133

Die Entwicklung des Exports an Industrieartikel . . . . . . . . . . . . . . . . . . 136

Das Problem der französischen industriellen Entwicklung . . . . . . . . . . . . . . 138

Vergleich der französischen und deutschen Inflationswirtschaft . . . . . . . . . . 138

Die französische Stabilisierungskrise . . . . . . . . . . . . . . . . . . . . 143

Die Probleme der französischen Stabilisierung . . . . . . . . . . . . . . . . . . .

Die Probleme der französischen Stabilisierung . . . . . . . . . . . . . . . . . . . . 144

Die Zahlungsbilanz Frankreichs . . . . . . . . . . . . . . . . . . . . . 145

Zusammenfassung und Perspektiven . . . . . . . . . . . . . . . . . . . . . . 147

IV. Die Vereinigten Staaten als führender imperialistischer Staat . . . . . . . . . . . . . . . 149

Die Elemente des Reichtums der Vereinigten Staaten . . . . . . . . . . . . . . . 150

Die amerikanische Landwirtschaft . . . . . . . . . . . . . . . . . . . . . 151

Der Kampf zwischen Farmern und industrieller Großbourgeoisie . . . . . . . . . . . 160

Die amerikanische Industrie . . . . . . . . . . . . . . . . . . . . . . . . . . 164

Versuch einer annähernden Bestimmung der Mehrwertsrate und Profitrate in der amerika-

nischen Industrie . . . . . . . . . . . . . . . . . . . . . . . . 169

Die Monopolorganisationen in der amerikanischen Industrie. . . . . . . . . . . 171

Das Vordringen des Bankkapitals . . . . . . . . . . . . . . . . . . . . . . . 173

Der Kapitalexport ． . . . . . . . . . . . . . . . . . . . . . . . . . . . . . 1777

Der amerikanische Imperialismus . . . . . . . . . . . . . . . . . . . . 182

V. Der Weg des italienischen Kapitalismus . . . . . . . . . . . . . . . . . . 186

Ungünstige und günstige Faktoren der Entwicklung des italienischen Kapitalismus . . . 186

Die Landwirtschaft Italiens . . . . . . . . . . . . . . . . . . . . . . . . 188

Die Entwicklung der italienischen Industrie . . . . . . . . . . . . . . . . 192

Die Entwicklung der italienischen Valuta . . . . . . . . . . . . . . . . . . . 198

Die Gestaltung des italienischen Staatshaushaltes . . . . . . . . . . . . . . . . . . . 199

Die Staatsschulden . . . . . . . . . . . . . . . . . . . . . . 200

Die interalliierten Schulden Italiens . . . . . . . . . . . . . . . . . . 201

Der Außenhandel Italiens . . . . . . . . . . . . . . . . . . . . . . . . . . 202

Die Zahlungsbilanz. . . . . . . . . . . . . . . . . . . . . . . 204

Die Wirtschaftspolitik des Faschismus . . . . . . . . . . . . . . . . 206

VI. Der japanische Imperialismus im Mittelpunkt der Weltpolitik . . . . . . . . . . . . . . 211

Entwicklung und spezielle Züge des japanischen Imperialismus . . . . . . . . . . . . 211

Die „Ubervölkerung“ Japans . . . . . . . . . . . . . . . . . . . . . . . . . . . 213

Die japanische Landwirtschaft und die Lage der Bauernschaft . . . . . . . . . . . . 214

Die Entwicklung der japanischen Industrie . . . . . . . . . . . . . . . . 216

Die Lage der industriellen Arbeiterschaft . . . . . . . . . . . . . . . . . 221

Der Außenhandel Japans . . . . . . . . . . . . . . . . . . . . . . . . . . . . 223

Das japanische Kolonialreich . . . . . . . . . . . . . . . . . . 225

Japan in der Weltwirtschaftskrise. . . . . . . . . . . . . . . . . . 226 
Der Kampf um China und die Interventionsgefahr . . . . . . . . . . . . . . . 233

Die militärische Schwäche Japans und die revolutionäre Bewegung . . . . . . . . . . . 242

VIERTER TEIL

Die Grofe Krise und ihre politischen Folgen. Wirtschaft und Politik 1928-1934 . . . . . . . . . . 250

Erstes Kapitel

Die ökonomischen Grundlagen der Wirtschaftskrisen . . . . . . . . . . . . . . . . . . . . . 251

Zweites Kapitel

Die Große Krise 1929-1933 . . . . . . . . . . . . . . . . . . . . . . . . . . . . . . . 261

Drittes Kapitel

Die Weltagrarkrise . . . . . . . . . . . . . . . . . . . . . . . . . . . . . . . . . . . . 284

Viertes Kapitel

Die Krise in den Kolonien . . . . . . . . . . . . . . . . . . . . . . . . . . . . . . . . . . 294

Fünftes Kapitel

Die erfolglosen Versuche zur künstlichen Überwindung der Krise . . . . . . . . . . . . . . . . 299

FÜNFTER TEIL

Neue Erscheinungen während der Weltwirtschaftskrise. . . . . . . . . . . . . . . . . . . . . . 308

Vorbemerkung . . . . . . . . . . . . . . . . . . . . . . . . . . . . . . . 309

I. Das allgemeine Bild der Krise. . . . . . . . . . . . . . . . . . . . . . . . . . . . . 311

II. Die Agrarkrise . . . . . . . . . . . . . . . . . . . . . . . . . . . . . . . . . . . . 336

III. Die Entwicklung der Krise . . . . . . . . . . . . . . . . . . . . . . . . . . . . . . . 351

IV. Die Lage der Arbeiterklasse und der Bauernschaft . . . . . . . . . . . . . . . . . . . . . . 375

Schlußbemerkung . . . . . . . . . . . . . . . . . . . . . . . . . . . . . 392

SECHSTER TEIL

Die kapitalistische Welt vor einer neuen Krise . . . . . . . . . . . . . . . . . . . . . . . . . . 395

I. Warum sind periodische Krisen im Kapitalismus unvermeidlich? . . . . . . . . . . . . . . 396

II. Der kapitalistische Markt und die Krisen . . . . . . . . . . . . . . . . . . . . . . . . . 398

III. Warum Zyklen und keine permanente Krise? . . . . . . . . . . . . . . . . . . . . . . . 402

IV. Warum wurden die Krisen tiefer und länger? . . . . . . . . . . . . . . . . . . . . . . . . 405

V. Die Krise von 1937 in den USA . . . . . . . . . . . . . . . . . . . . . . . . . . . . . 408

VI. Symptome der beginnenden Krise in anderen Ländern . . . . . . . . . . . . . . . . . . . 423

SIEBENTER TEIL

Die Wirtschaft des Kapitalismus im zweiten Weltkrieg . . . . . . . . . . . . . . . . . . . . . 429

I. Wirtschaftsregulierung und Planlosigkeit in den kapitalistischen Länder während des Krieges 431

II. Konzentration und Zentralisation der Produktion und des Kapitals während des Krieges . . . . 442

III. Rüstungswirtschaft und Technik . . . . . . . . . . . . . . . . . . . . . . . . . . . . 453 
IV. Die Inflation als Folge des Krieges . . . . . . . . . . . . . . . . . . . . . 463 ACHTER TEIL

„Das Kapital“ von Karl Marx und der moderne Kapitalismus ． . . . . . . . . . . . . . . . . 483

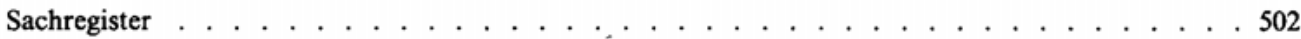

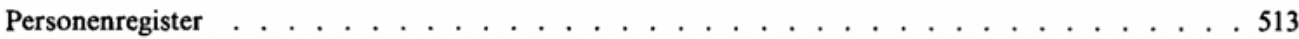




\section{Inhalt des dritten Bandes}

ERSTER TEIL

Der Kapitalismus des zwanzigsten Jahrhunderts . . . . . . . . . . . . . . . . . . . . . . . . . 1

Vorwort . . . . . . . . . . . . . . . . . . . . . . . . . . . . . . . . . 3

Kapitel I

Der Kapitalismus zu Beginn des zwanzigsten Jahrhunderts . . . . . . . . . . . . . . . . . . . 5

Kapitel II

Bis zum Ausbruch des ersten Weltkrieges. . . . . . . . . . . . . . . . . . . . . . . . . . . 17

Kapitel III

Die erste Etappe der allgemeinen Krise des Kapitalismus . . . . . . . . . . . . . . . . . . . . . . 22

Kapitel IV

Die zweite Etappe der allgemeinen Krise des Kapitalismus . . . . . . . . . . . . . . . . . . . 30

Kapitel $V$

Der Kapitalismus unserer Tage . . . . . . . . . . . . . . . . . . . . . . . . . . . . . . . 52

1. Die neue Lage in der Welt . . . . . . . . . . . . . . . . . . . . . . . . . . . . . . . . 52

2. Die wichtigsten ökonomischen Veränderungen innerhalb des Kapitalismus . . . . . . . . . . . 67

3. Die wichtigsten sozialen Veränderungen innerhalb des Kapitalismus . . . . . . . . . . . . . . 81

Kapitel VI

Die neue (dritte) Etappe der allgemeinen Krise des Kapitalismus . . . . . . . . . . . . . . . . . . 89

ZWEITER TEIL

Beiträge zu Problemen der politischen Ökonomiè des Kapitalismus . . . . . . . . . . . . . . . . 102

Vorwort . . . . . . . . . . . . . . . . . . . . . . . . . . . . 103

Der Marxismus und das ökonomische Grundgesetz des Kapitalismus . . . . . . . . . . . . . . 105

Zur Frage des bürgerlichen Staates . . . . . . . . . . . . . . . . . . . . . . . . . . . 123

Probleme des staatsmonopolistischen Kapitalismus . . . . . . . . . . . . . . . . . . . . . 135

Imperialistische Widersprüche und Krieg . . . . . . . . . . . . . . . . . . . . . . . . . . 152

Die Rolle der Bourgeoisie im nationalen Befreiungskampf der Völker . . . . . . . . . . . . . . 160

Das Problem der relativen Verelendung des Proletariats . . . . . . . . . . . . . . . . . . . 172

Die Frage der absoluten Verelendung . . . . . . . . . . . . . . . . . . . . . . . . . . . 179

Die Arbeiteraristokratie nach dem zweiten Weltkrieg . . . . . . . . . . . . . . . . . . . . . 189

Das Problem der Kapitalwanderung bei Ausgleichung der Durchschnittsprofitrate. Die Profitrate im

Monopolkapitalismus .. . . . . . . . . . . . . . . . . . . . . . . . . . . . 201

Das Volumen des kapitalistischen Marktes . . . . . . . . . . . . . . . . . . . . . . . . . . 217

Preisbildung, Inflation und Gold . . . . . . . . . . . . . . . . . . . . . . . . . . . . . 225

Veränderungen im zyklischen Verlauf der Reproduktion nach dem zweiten Weltkrieg . . . . . . . 248

Das Problem der Agrarkrisen. . . . . . . . . . . . . . . . . . . . . . . . . . . . . . . 273

Theoretische Probleme der Ökonomik des „Gemeinsamen Marktes“ . . . . . . . . . . . . . . . 308

Zu den Ursachen der Popularität des Keynesianismus . . . . . . . . . . . . . . . . . . . . . 322

Über die asiatische Produktionsweise . . . . . . . . . . . . . . . . . . . . . . . . . . . 341 
Anhang

Bibliographie der Veröffentlichungen Eugen Vargas . . . . . . . . . . . . . . 359

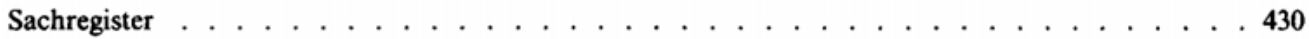

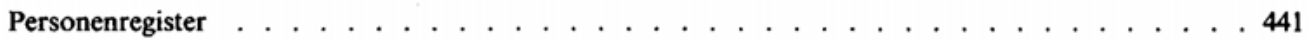

